\title{
Interview with Alessio Figalli
}

\author{
Ulf Persson (Chalmers University of Technology, Göteborg, Sweden), Editor of the EMS Newsletter
}

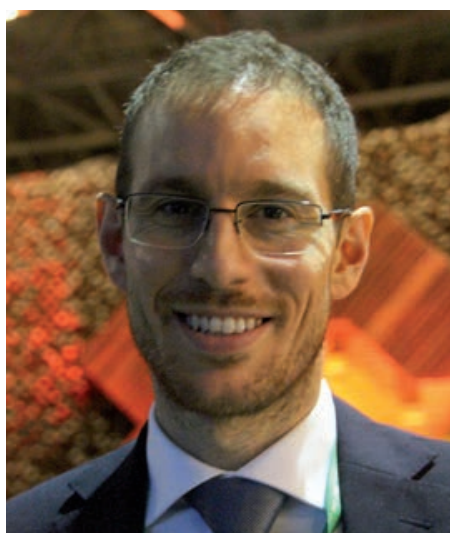

UP: Let me start with a standard and somewhat stupid question. Were you surprised getting the Fields Medal?

AF: (smiling) How could I answer anything but yes to that question? In fact, I even worried that it was a joke or a mix-up of some sort. But, to be serious though, in recent years, I tried not to think about it too much, lest I would be disappointed?

In other words, you ended up not being disappointed? I certainly am not.

Looking at your CV, so to speak, one gets the idea that you were not a child prodigy in mathematics because you entered university at a rather normal age of 18.

That is true. I really did not have any serious exposure to mathematics until I started university. I did not even know what a derivative was. In my last year of high school, I attended a local mathematics Olympiad and it was that which made me decide to apply to Scuola Normale. I attended the humanistic branch of high school, where you focus on the classics: Greek and Roman.

This is not too uncommon; many of my continental colleagues have the same background. Did you enjoy the classical education?

Some parts of it were nice of course (that is inevitable) but it was also a bit boring. Studying was not really fun; sports were my great interest as a teenager and as a child.

So you do not consider it a disadvantage coming to mathematics relatively late?

It was all fresh to me and exciting. Fellow students who had more exposure than me thought they knew everything and skipped classes and, in that way, they also missed something (in fact, much more than they realised). I, on the other hand, never missed a class and I did all the exercises.

You were a good student in other words - exemplary, in fact.

I was a good student.

How come you ended up doing analysis?

In fact, I was first fascinated by algebra. It struck me as so elegant. But I gained no real intuition; it all seemed like magic. It was actually when I took a course in functional analysis that I began really to be interested in analysis. There were so many neat proofs and simple but general arguments.

It is for this reason that functional analysis is referred to as soft analysis.

I do not agree.

And from functional analysis you move to harder and more technical analysis?

I was initially attracted by calculus of variations, which led me to PDEs. What I like about the subject is that you can draw pictures, that you can develop a visual intuition.

\section{No magic.}

No magic in the sense that you can understand what is going on, see things coming rather than have things thrown at you seemingly from nowhere. Of course, this was my very personal perspective.

Your career was fast. You got your PhD at 23, five years out of high school and, since then, it has been a straight road through French CNRS, a sojourn in the States and now at ETH. I guess Italian and French are your languages, in addition to ubiquitous English, but what about German?

I don't speak German yet but this is not really a problem. ETH is a very international environment so, in fact, not much German is spoken. The students are very good and the institution gives you very good support. It is close to an ideal environment.

\section{Do you read a lot and, if so, how do you read?}

I read, of course, a lot as a student. I was a good student and had to catch up quickly. I already started to read articles in my second year. I do not read articles linearly; I look for the good parts where something is actually "happening". Often, I do not bother with proofs but prefer to work it out for myself if I find something interesting. I am a fast reader and I go through a huge amount of articles, for my own work but also for evaluation - that is part of your duty.

It is often remarked that the way mathematics is written is not conducive to conveying mathematics, that precise statements and details tend to obscure the underlying ideas. The thoughts of a mathematician have to be encoded in strict, logical language, which therefore have to be decoded by the reader. A mathematical conversation is supposed to be the most efficient way.

Of course but it is a luxury. With mathematicians physically spread all over the world, you cannot have personal conversations with them all; you have to make do with papers and emails. 
How do you write your papers?

I never use paper and pen, except perhaps for doodling when I am thinking of mathematics. I type directly into the computer. Of course, I do not put down the definitive version right away; my proofs go through several revisions, during which I try to simplify arguments and rearrange material more naturally and logically. I can do it up to 50 times.

\section{All on the computer? But I guess you grew up with com- puters.}

I did. I am very comfortable with them.

But do you not need paper and pen when you manipulate formulas?

Yes, I do need paper and pen for that but I am also quite adept at doing complicated manipulations in my head. I do them all the time, also when typing.

Do you think that too many papers are being published? Definitely. There are too many bad papers or, rather, insignificant variations upon variations being written.

Most maths papers are never read, except possibly by the referee. They are not written to make a contribution to mathematics but to further the career of the authors to prove that they are active.

That could well be true and of course for students it is a very good exercise to write papers.

\section{But they should not be published?}

The screening process should definitely be more discriminatory. In fact, things are going much too fast nowadays. So much is produced and so much progress is being made. Things should cool down a bit; not so much should be thrown at you.

There are so many mathematicians nowadays so, unlike in the past, there are not enough good people to judge and form informed and personal opinions. So good young people may drown in a flood of mediocrity, while in the past they would have been spotted earlier - thus this emphasis on number of papers, modified by citations and prestige of journals in which they appear. This is good for bureaucrats by supplying a formal objective mechanism for evaluation, not taking into account contents. One particular measure I was told by younger colleagues was the h-index - in my generation we never had to worry about such things.

I know, of course, about the h-index. It is all very bad, I agree with you, and those indices can easily be doctored. On the other hand, it is something we just have to accept; it is unavoidable. We have to justify our existence; we cannot very well hope to be funded just because we are clever and beautiful. And those indices say something at least.

The problem is that those who pay us do not really care about what we are actually doing, as long as we are doing something.
It would be really bad for us if mathematicians had to justify themselves with practical applications. We should be grateful for this not being the case.

\section{You said things go too fast.}

Yes I did but this is exciting - so much progress. I would not like to have lived in the past because the foundations and techniques we now take for granted were not available to mathematicians then, and they had to struggle much more.

This reminds me of modern technology: people all the time using gadgets when they have no idea how they work. This makes for alienation. People in the past often manufactured their own implements and thus had a more intimate relation to them. A similar thing goes on in mathematics. To make progress and stay ahead, you need to use results you may not understand and, as a consequence, your relation to mathematics becomes much less intimate. Are you using results that you do not understand?

I guess I have to plead guilty on some accounts but I would say that it is minimal; once I am serious about something, even if I am only interested in citing a result, I do check all the details. It is part of the instinct of selfpreservation after all. No self-respecting mathematician would like to be caught making mistakes.

\section{Especially not a Fields Medallist.}

Especially not. I want to understand everything. And besides, the exact formulation of a theorem may not be precisely what you need. If you have grasped the ideas, you are in a position to be able to modify.

\section{Who are your mathematical heroes?}

That is easy: Caffarelli and de Giorgi. Caffarelli I met during my sojourn in the States and he has had a very deep impact on me, personally and scientifically. Many of the problems I work on were inspired by him. De Giorgi died relatively young so I only got to meet him through his papers, although I of course heard a lot about him at Scuola Normale, where he was a legend. He was of the opinion that mathematicians should only write in their own native language because that is the only language you can express yourself very well in...

\section{...but there is a problem of terminology...}

...I know. When it comes to mathematics, English is my native language. So when I give a talk to Italians, I hope that there will be some non-Italian in the audience so I can proceed in English. If not, you feel stupid lecturing in English. But back to de Giorgi. His papers are very different from the papers of today. There is much more text and verbal explanations and not so many formulas. Modern papers are much more technical.

So a paper by de Giorgi you would not want to skim? Ideally not but there is only so much time. Modern papers are more efficient. But he writes so beautifully and they are such a pleasure to read. 
You do not have any heroes of the past like Euler or Gauss? Did you ever read Bell's "Men of Mathematics"?

Frankly, I do not know that much of history. I am not that interested either. I am very happy working as a mathematician in the present, when there has been so much progress - maybe too much, as I said before. And as to the book you mention, I never read it but I suppose I should someday.

Frankly, I fear it is too late. It is, in a sense, a children's book, which, in many cases, has inspired young people to become mathematicians. But you are already a mathematician. So let us change tack - what is your position on the philosophy of mathematics, Platonism for example?

To be honest, I do not have any. It does not interest me or, at least, I have never given it a thought, which I guess amounts to the same thing. But I should say that one thing that attracts me very much about mathematics is that it is either true or false. So precise. Someone may not like my theorem but he cannot argue about its correctness. Whether he likes it or not, it is correct and that is something you do not have outside mathematics.
That is a philosophical statement if any. What about physics?

It is interesting but physical intuition I believe is different from mathematical.

Harder and maybe more subtle than in mathematics. People make elementary mistakes in reasoning about physics they would never do in mathematics.

No, I would not say that. It is just different.

And some of the intuition of those physicists has had remarkable impact on mathematics. But that is another story. Finally, what do you think this celebrity status will do to you?

Hopefully not too much. It is somewhat unreal at the moment. I went up very early the other morning and went through 600 emails - incidentally, it is thanks to this that I was able to respond to your email. This attention might be the most tangible effect of my celebrity status...

...it will pass soon. As a consequence, you must be very busy and I should not keep you any longer. Thanks for your time and a very nice conversation.

You are welcome. 